\title{
ON CONSTRAINED EQUATIONS WITH SINGULAR DIFFUSIVITY *
}

\author{
YOSHIKAZU GIGA ${ }^{\dagger}$ AND RYO KOBAYASHI ${ }^{\ddagger}$
}

\section{Dedicated to Professor Stanley Osher on the occasion of his 60th birthday}

1. Introduction. This is a continuation of our work $[\mathrm{KG}]$, $[\mathrm{GGK}]$, where we studied a gradient (flow) system of an energy whose energy density is not $C^{1}$ so that the diffusivity in the equation is very strong and its effect is even nonlocal. In this paper we consider the case when the values of unknowns are constrained. To be specific we consider a gradient (flow) system of the total variations of mappings with constraint of their values. Let us write the equation formally. For a mapping $u: \Omega \rightarrow \mathbf{R}^{N}$ with a domain $\Omega$ in $\mathbf{R}^{n}$ let $E_{1}(u)$ denote its total variation, i.e.,

$$
E_{1}(u)=\int_{\Omega}|\nabla u| d x .
$$

Let $\delta E_{1} / \delta u$ denote its 'first variation' (with respect to $L^{2}$ inner product). Then the unconstrained gradient system is formally written in the form

$$
u_{t}=-\delta E_{1} / \delta u
$$

for $u=u(x, t), x \in \Omega, t>0$, where $u_{t}$ denotes the time derivative, i.e., $u_{t}=\partial u / \partial t$. If the values of $u$ is constrained in some fixed (Riemannian) manifold $M$ embedded in $\mathbf{R}^{N}$, the first variation $\delta E_{1} / \delta_{M} u$ with this constraint is of the form

$$
\delta E_{1} / \delta_{M} u=P_{u}\left(\delta E_{1} / \delta u\right),
$$

where $P_{u}$ is the orthogonal projection to the tangent space of $M$ at the value of $u$. Thus our constrained gradient system is of the form

$$
u_{t}=-P_{u}\left(\delta E_{1} / \delta u\right) .
$$

The explicit form of (1.2) is

$$
u_{t}=\operatorname{div}\left(\frac{\nabla u}{|\nabla u|}\right) .
$$

If $M$ is a unit sphere $S^{N-1}$, then the explicit form of (1.3) is

$$
u_{t}=\operatorname{div}\left(\frac{\nabla u}{|\nabla u|}\right)+|\nabla u| u
$$

as explained in Example 2 in Section 2. An explicit calculation for (1.3) is for example in $[\mathrm{MSO}]$. Although the notion of solution of (1.4) is not a priori clear because of

\footnotetext{
*Received February 14, 2003; accepted for publication August 15, 2003.

${ }^{\dagger}$ Department of Mathematics, Hokkaido University, Sapporo 060-0810, Japan (gjr@math.sci.hokudai.ac.jp). Partly supported by the Grant-in-Aid for Scientific Research, No. 12874024, No. 14204011, the Japan Society for the Promotion of Science.

${ }_{\ddagger}$ Research Institute for Electronic Science, Hokkaido University, Sapporo 060-0812, Japan. Partly supported by the Grant-in-Aid for Scientific Research, No.13640095, the Japan Society for the Promotion of Science.
} 
singularity at $\nabla u=0$, a general nonlinear semigroup theory (initiated by Y. Kōmura [Ko]) applies under appropriate boundary conditions since the energy is convex. The theory yields the unique global solvability of the initial value problem for (1.2) under the Dirichlet boundary condition; see e.g. [Br], [Ba] and also [KG], [GGK], [HZ], $[\mathrm{ACM}],[\mathrm{ABCM}],[\mathrm{ABCM} 2],[\mathrm{BCM}]$... However, for (1.3) such a theory does not apply since it cannot be viewed as a gradient system of a convex functional. Even for smooth energy a constrained gradient system needs individual study for well-posedness. A typical example is the harmonic map flow equation. It is formally written in the form (1.3) where $E_{1}$ in (1.1) is replaced by the Dirichlet energy

$$
E_{2}(u)=\frac{1}{2} \int_{\Omega}|\nabla u|^{2} d x
$$

Its initial value problem is well-studied, for example, in $[\mathrm{ES}],[\mathrm{St}],[\mathrm{Cg}],[\mathrm{Ch}],[\mathrm{C}]$, [CDY], $[\mathrm{F}]$. The solution is independent of the way how $M$ is embedded in $\mathbf{R}^{N}$. For the gradient system of the total variation (1.3) even the notion of solution is unclear because of singularity at $\nabla u=0$.

In this paper, as a first attempt, we propose to formulate a constrained gradient system when the energy $\varphi$ is convex but having singularities by using subdifferentials $\partial \varphi$. It is formally written as

$$
u_{t} \in-P_{u}(\partial \varphi(u))
$$

The speed $u_{t}$ looks undetermined. However, under some regularity condition of $u$ we prove that the right derivative $d^{+} u / d t$ is uniquely determined. Like unconstrained problems it equals the minus of 'minimal section' of the convex set $P_{u}(\partial \varphi(u))$.

Unfortunately, even unique local solvability of the initial value problem for (1.3) is not clear. ${ }^{1}$ We restrict ourselves to consider piecewise constant initial data in a one dimensional domain — an open interval. We calculate the subdifferential $\partial \varphi$ when $\varphi$ is the total variation at a piecewise constant function. We further calculate the minimal section of $P_{u}(\partial \varphi(u))$ and construct a global solution for (1.3) with the Dirichlet condition by reducing the problem to a system of ordinary differential equations (ODEs). A key observation is that the minimal section is constant on each maximal spatial interval where the solution is constant so that the solution must stay as piecewise constant and the jump discontinuities are included in those of the initial data. This yields the uniqueness of a solution at least among piecewise constant functions. We say that each connected component of the graph of a piecewise constant function is a plateau.

We also study the behavior of solution when $M$ is the unit circle $S^{1}$. The equation of the motion of the plateau is presented, which is written in the form of reducing ODE. We identify the form of stationary solutions and prove that the solution becomes a stationary solution in finite time.

In the last part of this paper we demonstrate numerical simulations of the $S^{1}$ valued problem. Although our theoretical approach is restricted to the piecewise constant solutions at this point, our method also applies to calculating the evolution of the solutions in more general class. Our method to solve $S^{1}$-valued problem employs an angle variable, thus it is different from the method in [MSO] where $M$ is represented as a zero level set of some functions.

\footnotetext{
${ }^{1}$ Recently, a local solution is constructed for smooth initial data with small total variation under periodic boundary condition [GKY].
} 
Unlike the harmonic map flow, the notion of solution depends not only on $M$ itself, but also on the ambient space $\mathbf{R}^{N}$. Moreover, there are several ways to define the notion of total variation for mappings to $M$. The corresponding gradient system may differ. The definition of the total variation in this paper is not intrinsic; it depends on distance of the ambient space $\mathbf{R}^{N}$. For $S^{1}$-valued problem one is tempting to define the total variation of $u=(\cos \theta, \sin \theta)$ by $\int_{\Omega}|\nabla \theta| d x$. However, this energy is also singular when the jump of argument is $\pi$, so the dynamics starting with such jumps cannot be determined uniquely. There are several discussion to define the notion of mapping of bounded variation with values in $S^{1}$. In [GMS] a class of mappings approximated by smooth $S^{1}$ mappings was characterized.

Although there is huge literature on quasilinear parabolic equations with singularity at $\nabla u=0$, the singularity is weaker than ours in the sense that the diffusion effect is still local; see e.g. [D], [G]. There are several fields where equations with nonlocal singular diffusivity are proposed. The first example stems from material sciences for describing motion of antiphase grain boundaries [Gu]. In fact, a crystalline curvature flow equation was proposed $[\mathrm{AG}],[\mathrm{T}]$ as an example of anisotropic curvature flow equations $[\mathrm{G}],[\mathrm{Gu}]$ with singular interfacial energy. When the interface is a curve given as the graph of a function, a simple example is of the form (1.4) with $n=1$ [FG]. The second example stems from image analysis. In [ROF] it was proposed to use gradient flow system of the total variation with $L^{2}$-constraint for a grey level function $u$ to remove noises from images. The third example stems from plasticity problem [HZ]. The fourth example is derived from the phase field model of grain structure evolution which include grain boundary migrations and grain rotation $[\mathrm{KWC}],[\mathrm{WKC}],[\mathrm{LW}],[\mathrm{GBP}]$. The equation of orientation with singular diffusivity is coupled with the equation of ordering parameter. This model yields a mathematical subproblem with spatially non-uniform energy. We developed a mathematical theory which handles such a non-uniform equation with singular diffusivity in $[\mathrm{KG}]$ and [GGK] together with the case of the uniform energy. By now well-posedness for unconstrained gradient system (1.3) is established by many authors [FG], [HZ], [ACM], $[\mathrm{ABCM}],[\mathrm{ABCM} 2],[\mathrm{ChW}] \ldots$

Although the curvature flow equations with singular diffusivity do not have the divergence structure of the form (1.2), they are well-studied for evolution of curves [GG1] based on order-preserving structure. For a surface evolution the corresponding theory is widely open; see e.g. [BN], [GPR]. There are several other applications of singular diffusivity, for example for formation of shocks of conservation laws [GG2], [TGO].

The problem with value constraint is proposed by [TSC] in image processing to remove noise from chromaticity - direction field of color gray-level mappings $u=$ $\left(u_{1}, u_{2}, u_{3}\right)$ keeping its strength $u_{1}^{2}+u_{2}^{2}+u_{3}^{2}=1$. There is a nice book for background of the problems form image processing. As mentioned in $[\mathrm{S}, \S 6.3]$ the well-posedness for the initial-boundary problem for constrained problem (1.3) has not yet been studied even for (1.5). This type of constrained problems also naturally arise in multi-grain problems $[\mathrm{KWC}]$ where $u$ is an angle of averaged crystallographical directions.

2. Gradient system with constraint. We prepare an abstract framework for studying gradient systems of a convex functional. Let $\varphi(\not \equiv \infty)$ be a convex, lower semicontinuous function on a Hilbert space $H$ with values in $\mathbf{R} \cup\{\infty\}$. The gradient system for $\varphi$ is of the form

$$
\frac{d u}{d t}(t) \in-\partial \varphi(u(t)) \quad \text { for } \quad t>0,
$$


where $\partial \varphi(v)$ denotes the subdifferential of $\varphi$ at $v$, i.e.,

$$
\partial \varphi(v):=\{\xi \in H ; \varphi(v+h)-\varphi(v) \geq\langle h, \xi\rangle \text { for all } h \in H\}
$$

and $u$ is a function from $(0, \infty)$ to $H$. It is well known (see e.g. [Br], [Ba]) that the problem (2.1) admits a unique global solution for any given initial data in $H$. We next consider a gradient system with constraints on values of functions. Let $(v, w)$ denote the standard inner product of $v, w \in \mathbf{R}^{N}$. Let $\Omega$ be a smooth, bounded domain in $\mathbf{R}^{n}$. The space of $\mathbf{R}^{N}$-valued square integrable functions is denoted by $L^{2}\left(\Omega ; \mathbf{R}^{N}\right)$. As a Hilbert space $H$ we take $L^{2}\left(\Omega ; \mathbf{R}^{N}\right)$ equipped with the inner product

$$
\langle f, g\rangle=\int_{\Omega}(f(x), g(x)) d x \quad \text { for } \quad f, g \in H .
$$

Let $M$ be a smoothly embedded complete manifold in $\mathbf{R}^{N}$. For a given point $v \in M$ let $\pi_{v}$ denote the orthogonal projection from $\mathbf{R}^{N}=T_{v} \mathbf{R}^{N}$ to the tangent space $T_{v} M$ of $M$ at $v$. Let $\mathcal{M}$ be the space of $L^{2}$-mappings from $\Omega$ to $M$ i.e.,

$$
\mathcal{M}=\{f \in H ; f(x) \in M \quad \text { for } \quad \text { a.e. } x \in \Omega\} .
$$

For $g \in \mathcal{M}$ we define a mapping from $H$ to $H$ by

$$
P_{g}(f)(x)=\pi_{g(x)}(f(x)) \quad \text { for } \quad \text { a.e. } x \in \Omega,
$$

where $f \in H$. By definition $P_{g}$ is an orthogonal projection of $H$ so that its image $H_{g}$ is a closed subspace of $H$. (Actually, it is the tangent space of the Hilbert manifold $\mathcal{M}$ at $g$.)

A constrained (by $M$ ) gradient systems is of the form

$$
\frac{d u}{d t}(t) \in-P_{u(t)}(\partial \varphi(u(t))) \text { for } t>0 .
$$

This problem is no longer dissipative so unique globally solvability is not expected even if $\varphi$ is smooth so that no singular diffusivity appears. In fact, there is a counterexample for global solvability of a smooth solution and uniqueness for the harmonic map flow in Example 1.

EXAMPLE 1 (Harmonic map flow). Let $g$ be a Lipschitz map from $\partial \Omega$ to $M$. For $v \in H$ we set

$$
\varphi(v)= \begin{cases}\frac{1}{2} \int_{\Omega}|\nabla v|^{2} d x, & \text { if } \partial_{x_{i}} v, v \in H(1 \leq i \leq n) \text { with } v=g \text { on } \partial \Omega \\ +\infty, & \text { otherwise. }\end{cases}
$$

Then (2.2) is the harmonic map flow equation with the Dirichlet condition $v=g$ on $\partial \Omega$. Here $\nabla v=\left(\partial_{x_{1}} v, \ldots, \partial_{x_{n}} v\right)$ and $\partial_{x_{i}}=\partial / \partial x_{i}$ and $|\nabla v|^{2}$ denotes the sum of all squares of $\partial_{x_{i}} v^{s}$ for $v=\left(v^{1}, \ldots, v^{N}\right)$. Unconstrained problem (2.1) for this $\varphi$ is the heat equation with the Dirichlet condition. Of course, $\varphi$ is a lower semicontinuous, convex function in $H$.

The harmonic map flow equation is well-studied by many authors. Uniqueness and global solvability depend on the dimension of $\Omega$ and also geometric properties of manifold $M$. For example if $\Omega$ is two-dimensional, i.e., $n=2$, there is a unique global weak solution which is regular except a finite number of isolated points and 
the energy is decreasing in time $[\mathrm{St}],[\mathrm{Cg}],[\mathrm{F}]$. When $n \geq 3$, although there exists a global weak solution, it may not be unique [Ch], [C]. If $M=S^{1}$, then the global solution is smooth. However, if $M=S^{2}$, there exists a smooth local solution which develops singularities in finite time [CDY] when $\Omega$ is a two dimensional disk. See, for example, $[\mathrm{S}]$ for more complete list of references on this topics.

If

$$
M=S^{N-1}=\left\{w \in \mathbf{R}^{N} ;|w|=1\right\}
$$

i.e. $M$ is the unit sphere, then for $z \in M$

$$
\pi_{z}(y)=y-(y, z) z \quad \text { for } \quad y \in \mathbf{R}^{N} .
$$

Since $\partial \varphi(v)=\{-\Delta v\}$ for $v$ (belonging to the domain of $\partial \varphi$ ),

$$
-P_{v}(\partial \varphi(v))=\{\Delta v-(\Delta v, v) v\} .
$$

Since $|v|=1$ so that $(\Delta v, v)=\operatorname{div}(\nabla v, v)-|\nabla v|^{2}=-|\nabla v|^{2}$, we observe that

$$
-P_{v}(\partial \varphi(v))=\left\{\Delta v+|\nabla v|^{2} v\right\} .
$$

So $(2.2)$ is formally written as

$$
\frac{\partial u}{\partial t}=\Delta u+|\nabla u|^{2} u
$$

EXAMPLE 2 (Total variation flow with constraint). Let $g$ be a Lipschitz map form $\partial \Omega$ to $M$. Let $\tilde{g}$ denote a Lipschitz extension of $g$ to $\mathbf{R}^{n}$. For $v \in H$ let $\tilde{v}$ be its extension to $\mathbf{R}^{n}$ such that $\tilde{v}(x)=\tilde{g}(x)$ for $x \in \mathbf{R}^{n} \backslash \Omega$. We set

$$
\varphi(v)= \begin{cases}\int_{\bar{\Omega}}|\nabla \tilde{v}(x)| d x, & \text { if } \tilde{v} \in B V\left(\Omega ; \mathbf{R}^{N}\right) \\ +\infty, & \text { otherwise, }\end{cases}
$$

where $B V$ denotes the space of functions of bounded total variation. The quantity $\varphi(v)$ is the total variation of the measure $\nabla v$ in $\mathbf{R}^{n}$. The reason we extend $v$ to $\tilde{v}$ is that we would rather measure the discrepancy of $v$ from $g$ on the boundary. By this choice of $\varphi(2.1)$ is the total variation flow equation with Dirichlet condition. Its formal form is

$$
\frac{\partial u}{\partial t}=\operatorname{div}\left(\frac{\nabla u}{|\nabla u|}\right)
$$

It is easy to see that $\varphi$ is a convex, lower semicontinuous function in $H$ [GGK]. The equation (2.2) is the Dirichlet problem for the total variation flow equation with constraint. If $M$ is the unit sphere (2.3), then its formal form is

$$
\frac{\partial u}{\partial t}=\operatorname{div}\left(\frac{\nabla u}{|\nabla u|}\right)+|\nabla u| u
$$

since $\left(\operatorname{div}\left(\frac{\nabla v}{|\nabla v|}\right), v\right)=\operatorname{div}\left(\frac{\nabla v}{|\nabla v|}, v\right)-|\nabla v|=-|\nabla v|$ for $v$ satisfying $|v|=1$. 
EXAmPLE 3 (A simple inhomogeneous example). Let $a$ be a positive continuous function in $\bar{\Omega}$. Instead of Example 2 we set

$$
\varphi(v)=\int_{\bar{\Omega}} a(x)|\nabla \tilde{v}(x)| d x
$$

for $v \in B V\left(\Omega, \mathbf{R}^{N}\right)$ and $\varphi(v)=+\infty$ otherwise. This $\varphi$ is also a convex, lower semicontinuous function in $H$. This type of inhomogeneous version is important in application to multi-grain problems [GGK], $[\mathrm{KG}]$ and also image processing e.g. $[\mathrm{ChW}]$.

3. Characterization of speed. The evolution laws (2.1) and (2.2) look ambiguous since $\partial \varphi$ is multivalued. Like (2.1) the speed $d u / d t$ of the evolution by $(2.2)$ is actually uniquely determined under stronger assumptions than those for (2.1). We state such a characterization of the speed in this section. Unfortunately, it does not yield the uniqueness of a solution of the initial value problem for (2.2).

We prepare several notations. For a closed convex set $A$ in a Hilbert space there exists a unique point $z$ closest to the origin. We shall write $z$ by ${ }^{0} A$. Since $\partial \varphi(v)$ is always a closed convex set in $H,{ }^{0}(\partial \varphi(v))$ is well-defined and is denoted by $\partial^{0} \varphi(v)$. It is called the canonical restriction (or minimal section) of $\partial \varphi(v)$. The set $P_{v}(\partial \varphi(v))$ is also a convex set in $H_{v}$ for $v \in \mathcal{M}$ since $P_{v}$ is an orthogonal projection. However, it may not be closed. If there exists a point $z^{\prime} \in P_{v}(\partial \varphi(v))$ which is closest to the origin of $H_{v}$, it must be unique since the set is convex. We shall denote $z^{\prime}$ by ${ }^{0} P_{v}(\partial \varphi(v))$. We call this element the minimal section (of $P_{v}(\partial \varphi(v))$.

Theorem 3.1. Assume that $\delta>0$ and that $M$ is compact. Assume that $u$ : $\left[t_{0}, t_{0}+\delta\right] \rightarrow \mathcal{M} \subset H$ is continuous and right differentiable. Assume that the right derivative $d^{+} u / d t$ is continuous in $\left[t_{0}, t_{0}+\delta\right]$ and that

$$
\left\{\partial^{0} \varphi\left(u(t)+P_{u(t)}(u(t+\tau)-u(t))\right) ; t, t+\tau \in\left[t_{0}, t_{0}+\delta\right], \tau \in \mathbf{R}\right\}
$$

is bounded in $H$. If $u$ satisfies

$$
\frac{d^{+} u}{d t}(t) \in-P_{u(t)}\left(\partial \varphi(u(t)) \quad \text { for } \quad t \in\left[t_{0}, t_{0}+\delta\right),\right.
$$

then

$$
\frac{d^{+} u}{d t}(t)=-{ }^{0} P_{u(t)}\left(\partial \varphi(u(t)) \quad \text { for } \quad t \in\left[t_{0}, t_{0}+\delta\right) .\right.
$$

In particular, the minimal section of $-P_{u(t)}(\partial \varphi(u(t)))$ always exists for $t \in\left[t_{0}, t_{0}+\delta\right)$.

Proof. It suffices to prove (3.2) for $t=t_{0}$. We may assume that $t_{0}=0$. We set

$$
h(s)=u(s)-u(0), \quad P_{s}=P_{u(s)} \quad \text { for } \quad s \in[0, \delta)
$$

to simplify the notation. By (3.1)

$$
\left\langle\frac{d^{+} u}{d t}(s), h(s)\right\rangle=\left\langle-\frac{d^{+} u}{d t}(s),-P_{s} h(s)\right\rangle \leq \varphi\left(u(s)-P_{s} h(s)\right)-\varphi(u(s)) .
$$

By definition for $\xi \in P_{0}(\partial \varphi(u(0))$ we have

$$
\langle-\xi, h(s)\rangle=\left\langle-\xi, P_{0} h(s)\right\rangle \geq \varphi(u(0))-\varphi\left(u(0)+P_{0} h(s)\right) .
$$


Combining (3.3) and (3.4), we obtain

$$
\left\langle\frac{d^{+} u}{d t}(s), h(s)\right\rangle \leq\langle-\xi, h(s)\rangle+\Phi(s)+\Psi(s)
$$

with

$$
\begin{aligned}
& \Phi(s)=\varphi\left(u(s)-P_{s} h(s)\right)-\varphi(u(0)), \\
& \Psi(s)=\varphi\left(u(0)+P_{0} h(s)\right)-\varphi(u(s)) .
\end{aligned}
$$

We divide both hand sides by $s>0$. Sending $s$ to zero yields

$$
\left\|\frac{d^{+} u}{d t}(0)\right\|^{2} \leq\left\langle-\xi, \frac{d^{+} u}{d t}(0)\right\rangle \leq\|\xi\|\left\|\frac{d^{+} u}{d t}(0)\right\|
$$

if we admit

$$
\varlimsup_{s \downarrow 0} \Phi(s) / s=0 \quad \text { and } \quad \varlimsup_{s \downarrow 0} \Psi(s) / s=0,
$$

where $\|\cdot\|$ denotes the norm in $H$. By (3.5) we observe that $d^{+} u(0) / d t$ is the minimal section of $P_{0}(\partial \varphi(u(0))$.

It remains to prove (3.6). We shall present the proof for $\Phi$ since the proof for $\Psi$ is similar. By definition of subdifferentials

$$
\varphi\left(u(s)-P_{s} h(s)\right)-\varphi(u(0)) \leq\left\langle\left(I-P_{s}\right) h(s), \partial^{0} \varphi\left(u(s)-P_{s} h(s)\right)\right\rangle
$$

By our boundedness assumption on $\partial^{0} \varphi$ it suffices to prove that

$$
\lim _{s \downarrow 0}\left\|\left(I-P_{s}\right) h(s)\right\| / s=0 .
$$

By definition of the tangent space there exists a constant $C$ and $\delta_{0}>0$ that satisfies

$$
\left|\left(I-\pi_{v}\right) \zeta\right| \leq C\left|\pi_{v} \zeta\right|^{2}
$$

for all $\zeta \in \mathbf{R}^{N}, v \in M$ satisfying $\zeta+v \in M$ and $|\zeta| \leq \delta_{0}$. (Note that $\delta_{0}$ can be taken independent of $v$ since $M$ is compact.) Indeed, for small $\delta>0$ there is a ball $B_{\delta}(v)$ of radius $\delta$ centered at $v$ such that $M$ is expressed as the graph of function $x_{N}=f\left(x^{\prime}\right)$ in $B_{\delta}(v)$ with $f\left(v^{\prime}\right)=v_{N}, \nabla^{\prime} f\left(v^{\prime}\right)=0$ by a rotation, where $x=\left(x^{\prime}, x_{N}\right), v=\left(v^{\prime}, v_{N}\right)$ and $\nabla^{\prime}=\nabla_{x^{\prime}}$. Since $M$ is smooth, we observe that $f\left(v^{\prime}+\zeta^{\prime}\right)-f\left(v^{\prime}\right)=O\left(\left|\zeta^{\prime}\right|^{2}\right)$ as $\left|\zeta^{\prime}\right| \rightarrow 0$. This implies that $\left|\zeta_{N}\right| \leq C\left|\zeta^{\prime}\right|^{2}$ for small $\zeta=\left(\zeta^{\prime}, \zeta_{N}\right)$ which yields (3.8).

For $\delta \in\left(0, \delta_{0}\right)$ we set

$$
\Omega_{s \delta}=\{x \in \Omega|| h(x, s) \mid \geq \delta\}
$$

and denote its complement in $\Omega$ by $\Omega_{s \delta}^{c}$. By (3.8) we have

$$
\int_{\Omega_{s \delta}^{c}} \frac{1}{s^{2}}\left|\left(I-P_{s}\right) h(s)\right|^{2} d x \leq C \delta^{2} \int_{\Omega} \frac{|h(s)|^{2}}{s^{2}} d x
$$

and

$$
\begin{aligned}
\int_{\Omega_{s \delta}} & \frac{1}{s^{2}}\left|\left(I-P_{s}\right) h(s)\right|^{2} d x \leq \int_{\Omega_{s \delta}} \frac{1}{s^{2}}|h(s)|^{2} d x \\
\leq & \leq\left[\left(\int_{\Omega}\left|\frac{h(s)}{s}-\frac{d^{+} u(0)}{d t}\right|^{2} d x\right)^{1 / 2}+\left(\int_{\Omega_{s \delta}}\left|\frac{d^{+} u}{d t}(0)\right|^{2} d x\right)^{1 / 2}\right]^{2} .
\end{aligned}
$$


Since $h(s) / s \rightarrow d^{+} u(0) / d t$ as $s \downarrow 0$ in $H,(3.9)$ and (3.10) yield

$$
\varlimsup_{s \downarrow 0}\left\|\left(I-P_{s}\right) h(s)\right\|^{2} / s^{2} \leq C \delta^{2} \int_{\Omega}\left|\frac{d^{+} u}{d t}(0)\right|^{2} d x+\varlimsup_{s \downarrow 0} \int_{\Omega_{s \delta}}\left|\frac{d^{+} u}{d t}(0)\right|^{2} d x .
$$

Since $h(s) \rightarrow 0$ in $H$, the Lebegue measure of $\Omega_{s \delta}$ tends to zero by taking a subsequence. Thus

$$
\varlimsup_{s \downarrow 0}\left\|\left(I-P_{s}\right) h(s)\right\|^{2} / s^{2} \leq C \delta^{2} \int_{\Omega}\left|\frac{d^{+} u}{d t}(0)\right|^{2} d x .
$$

Sending $\delta$ to zero yields (3.7). We now obtain (3.6).

4. One dimensional piecewise constant evolution. We now consider the total variation flow with constraint (Example 2) when the domain $\Omega$ is an interval $\left(z_{0}, z_{1}\right)$. We consider the initial value problem

$$
\frac{d u}{d t}(t) \in-P_{u(t)}(\partial \varphi(u(t))),\left.u\right|_{t=0}=u_{0}
$$

with $\varphi$ defined by $(2.4)$ with $\Omega=\left(z_{0}, z_{1}\right)$. We consider a piecewise constant initial data

$$
u_{0}(x)=h_{i}^{0} \in \mathbf{R}^{N} \quad \text { on } \quad\left(x_{i}, x_{i+1}\right), i=0,1, \ldots, \ell-1, \ell \geq 2,
$$

where $z_{0}=x_{0}<x_{1}<x_{2}<\cdots<x_{\ell}=z_{1}$. The boundary values $h_{0}^{0}, h_{\ell-1}^{0}$ are taken so that $h_{0}^{0}=g\left(z_{0}\right)$ and $h_{\ell-1}^{0}=g\left(z_{1}\right)$. We also assume that $h_{i}^{0} \neq h_{i+1}^{0}$ for $i=0,1, \ldots, \ell-2$.

We shall seek a solution $u(t)=u(x, t)$ of $(4.1)-(4.2)$ when $u(x, t)$ is piecewise constant and its jump discontinuities are included in $\left\{x_{i}\right\}_{i=1}^{\ell-1}$.

4.1. Subdifferentials. We first calculate the subdifferential $\partial \varphi$ of $\varphi$ defined by (2.4) at a piecewise linear function $u_{0}$ defined by (4.2). We set

$$
m_{i}^{0}=\left(h_{i}^{0}-h_{i-1}^{0}\right) /\left|h_{i}^{0}-h_{i-1}^{0}\right|, i=1, \ldots, \ell-1 .
$$

LEMMA 4.1 Let $f \in L^{2}\left(\Omega ; \mathbf{R}^{N}\right)$ be of the form

$$
f(x)=-(\xi(x))_{x},|\xi(x)| \leq 1, x \in \Omega=\left(z_{0}, z_{1}\right)
$$

for some continuous $\xi$ in $\Omega$ that satisfies

$$
\xi\left(x_{i}\right)=m_{i}^{0}, i=1,2, \ldots, \ell-1 .
$$

Then $f \in \partial \varphi\left(u_{0}\right)$. Conversely, if $f \in \partial \varphi\left(u_{0}\right)$, then $f$ is of the form (4.4) with (4.5). Here ()$_{x}$ denotes derivative in the sense of distributions.

Proof. The proof is similar to that of [GGK, $§ 3.2$, Lemma 1]. We shall check

$$
\left\langle v-u_{0}, f\right\rangle \leq \varphi(v)-\varphi\left(u_{0}\right)
$$

for all $v \in D(\varphi)=\{v ; \varphi(v)<\infty\}$. By definition

$$
\left\langle v-u_{0}, f\right\rangle=-\int_{\Omega}\left(v-u_{0}, \xi_{x}\right) d x .
$$


Since $|\xi| \leq 1$, integrating by parts we see

$$
-\int_{\Omega}\left(v, \xi_{x}\right) d x=\int_{\Omega}\left(v_{x}, \xi\right) d x-\left.\left(u_{0}, \xi\right)\right|_{z_{0}} ^{z_{1}} \leq \varphi(v)-\left.\left(u_{0}, \xi\right)\right|_{z_{0}} ^{z_{1}},
$$

where $v_{x}$ is regarded as a Radon measure; $\varphi(v)$ equals the total variation of $(\tilde{v})_{x}$. For example

$$
\varphi\left(u_{0}\right)=\sum_{i=1}^{\ell-1}\left|h_{i}^{0}-h_{i-1}^{0}\right| .
$$

Since $\xi\left(x_{i}\right)=m_{i}$, we see that

$$
\begin{aligned}
\int_{\Omega}\left(u_{0}, \xi_{x}\right) d x & =\left.\left(u_{0}, \xi\right)\right|_{z_{0}} ^{z_{1}}-\sum_{i=1}^{\ell-1}\left(h_{i}^{0}-h_{i-1}^{0}\right) m_{i}^{0} \\
& =\left.\left(u_{0}, \xi\right)\right|_{z_{0}} ^{z_{1}}-\varphi\left(u_{0}\right) .
\end{aligned}
$$

The formula (4.6)-(4.8) now yields

$$
\begin{aligned}
\left\langle v-u_{0}, f\right\rangle & \leq \varphi(v)-\left.\left(u_{0}, \xi\right)\right|_{z_{0}} ^{z_{1}}+\left.\left(u_{0}, \xi\right)\right|_{z_{0}} ^{z_{1}}-\varphi\left(u_{0}\right) \\
& =\varphi(v)-\varphi\left(u_{0}\right)
\end{aligned}
$$

which implies $f \in \partial \varphi\left(u_{0}\right)$.

Conversely, assume that $f \in \partial \varphi\left(u_{0}\right)$. Let $\zeta$ be a primitive of $-f$. Since $f \in$ $L^{2}\left(\Omega ; \mathbf{R}^{N}\right), \zeta$ must be absolutely continuous on $\Omega$. The condition $f \in \partial \varphi\left(u_{0}\right)$ is equivalent to

$$
\int_{\Omega}\left(v-u_{0}, \zeta_{x}\right) d x \leq \varphi(v)-\varphi\left(u_{0}\right) .
$$

We test various $v$ in this inequality to derive properties of $\zeta$.

We plug

$$
v(x)=u_{0}(x)-\lambda m_{i} \int_{z_{0}}^{x} \delta\left(\tau-x_{i}\right) d \tau, \lambda \in \mathbf{R},|\lambda|<\left|h_{i}^{0}-h_{i-1}^{0}\right|
$$

in (4.9) and integrate by parts to get

$$
-\lambda\left(m_{i}, \zeta\left(x_{i}\right)\right) \leq-\lambda .
$$

for $i=1, \ldots, \ell-1$. Here $\delta$ denotes the Dirac delta function. Since this inequality holds for both positive and negative $\lambda$, we conclude that

$$
\left(m_{i}, \zeta\left(x_{i}\right)\right)=1, i=1, \ldots, \ell-1 .
$$

For $\hat{x} \in\left(z_{0}, z_{1}\right) \backslash\left\{x_{i}\right\}_{i=1}^{\ell-1}$ we set

$$
v(x)=u_{0}(x)+\lambda \int_{z_{0}}^{x} m \delta(\tau-\hat{x}) d \tau, \lambda \in \mathbf{R}, m \in S^{N-1} .
$$


We plug this $v$ in (4.9) and integrate by parts to get

$$
\lambda(m, \zeta(\hat{x})) \leq|\lambda|
$$

Since this inequality holds for both positive and negative $\lambda$, we observe that

$$
|(m, \zeta(\hat{x}))| \leq 1
$$

Since $m \in S^{N-1}$ is arbitrary, this implies $\left.\mid \zeta(\hat{x})\right) \mid \leq 1$. Be continuity of $\zeta$ we see that

$$
|\zeta(x)| \leq 1 \quad \text { for all } \quad x \in \Omega \text {. }
$$

Since $\left(m_{i}, \zeta\left(x_{i}\right)\right)=1$, the inequality $|\zeta(x)| \leq 1$ implies that $\zeta\left(x_{i}\right)=m_{i}$. We have thus proved that $f \in \partial \varphi\left(u_{0}\right)$ must have the form (4.4)-(4.5).

4.2. Minimal section. We shall calculate ${ }^{0} P_{u_{0}} \partial \varphi\left(u_{0}\right)$ for a piecewise constant function $u_{0}$ in (3.7). In general it is not clear that ${ }^{0} P_{v} \partial \varphi(v)=P_{v} \partial^{0} \varphi(v)$ but for our $u_{0}$ this property holds.

Lemma 4.2. Let $L_{i}$ be the length of the interval $\left(x_{i}, x_{i+1}\right)$, i.e., $L_{i}=x_{i+1}-x_{i}$. Then

$$
-{ }^{0} P_{u_{0}}\left((\partial \varphi)\left(u_{0}\right)\right)(x)=\left\{\begin{array}{lll}
L_{i}^{-1} \pi_{h_{i}^{0}}\left(m_{i+1}^{0}-m_{i}^{0}\right) & \text { for } \quad \begin{array}{l}
x \in\left(x_{i}, x_{i+1}\right), \\
0
\end{array} & \text { for } \left.\quad \begin{array}{l}
i=1, \ldots, \ell-2, \\
0
\end{array} \quad x x_{0}, x_{1}\right) \cup\left(x_{\ell-1}, x_{\ell}\right) .
\end{array}\right.
$$

Moreover, $\left.{ }^{0} P_{u_{0}}\left((\partial \varphi) u_{0}\right)=P_{u_{0}}\left(\partial^{0} \varphi\right)\left(u_{0}\right)\right)$.

Proof. By Lemma 4.1 we already know the explicit form of $\partial \varphi\left(u_{0}\right)$. If $q=$ ${ }^{0} P_{u_{0}}(\partial \varphi)\left(u_{0}\right)$, it must be

$$
q=-P_{u_{0}}\left(\eta_{x}\right)
$$

with $\eta$ minimizing

$$
\|q\|^{2}=\sum_{i=0}^{\ell-1} \int_{x_{i}}^{x_{i+1}}\left|\pi_{h_{i}^{0}} \eta_{x}\right|^{2} d x
$$

with constraints $\eta\left(x_{i}\right)=m_{i}^{0}(i=1,2, \ldots, \ell-1)$ and $|\eta(x)| \leq 1$ for $x \in \Omega$. It suffices to minimize

$$
\int_{x_{i}}^{x_{i+1}}\left|\pi_{h_{i}^{0}} \eta_{x}\right|^{2} d x
$$

with above constraint. The answer is easy. The minimum is attained when $\eta$ is linear

$$
\eta(x)=\left\{\left(x-x_{i}\right) m_{i+1}^{0}+\left(x_{i+1}-x\right) m_{i}^{0}\right\} L_{i}^{-1} \quad \text { for } \quad x \in\left(x_{i}, x_{i+1}\right)
$$

for $i=1,2 \ldots, \ell-1$ and

$$
\eta(x)=\left\{\begin{array}{l}
m_{1}^{0} \quad \text { for } \quad x \in\left(x_{0}, x_{1}\right) \\
m_{\ell-1}^{0} \quad \text { for } \quad x \in\left(x_{\ell-1}, x_{\ell}\right) .
\end{array}\right.
$$

Since $q=-P_{u_{0}}\left(\eta_{x}\right)$, we have an expression of ${ }^{0} P_{u_{0}}(\partial \varphi)\left(u_{0}\right)$ in Lemma 4.2.

Since $\partial^{0} \varphi\left(u_{0}\right)$ is also computable and

$$
\partial^{0} \varphi\left(u_{0}\right)= \begin{cases}L_{i}^{-1}\left(m_{i+1}^{0}-m_{i}^{0}\right) & \text { for } \quad x \in\left(x_{i}, x_{i+1}\right), i=1,2, \ldots, \ell-2, \\ 0 & \text { for } \quad x \in\left(x_{0}, x_{1}\right) \cup\left(x_{\ell-1}, x_{\ell}\right),\end{cases}
$$

we obtain ${ }^{0} P_{u_{0}}(\partial \varphi)\left(u_{0}\right)=P_{u_{0}}\left(\partial^{0} \varphi\left(u_{0}\right)\right)$. 
4.3. Dynamics. We consider (4.1)-(4.2) assuming that

$$
u(x, t)=h_{i}(t) \in \mathbf{R}^{N} \quad \text { on } \quad\left(x_{i}, x_{i+1}\right), i=0,1, \ldots, \ell-1, t>0
$$

with $h_{0}(t)=g\left(z_{0}\right)$ and $h_{\ell-1}(t)=g\left(z_{1}\right)$. The values $h_{i}(t)$ and $h_{i+1}(t)$ may agree for some $t>0$. It turns out that the problem (4.1)-(4.2) is reduced to an ODE system for $h_{i}$ 's. Moreover, there exists a unique global solution.

Theorem 4.3. Assume that $M$ is compact. There exists a unique

$$
h(t)=\left(h_{1}(t), \ldots, h_{\ell-2}(t)\right)
$$

such that $h_{i}(1 \leq i \leq \ell-2)$ is Lipschitz continuous from $[0, \infty)$ to $M$ which is smooth except finitely many points such that (4.10) solves (4.1)-(4.2). Moreover, $h_{i}$ solves

$$
\frac{d h_{i}(t)}{d t}=\frac{1}{L_{i}} \pi_{h_{i}(t)}\left(m_{i+1}(t)-m_{i}(t)\right) \quad \text { for } \quad \begin{aligned}
& x \in\left(x_{i}, x_{i+1}\right), \\
& i=1,
\end{aligned}
$$

for sufficiently small $t>0$, where

$$
m_{i}(t)=\left(h_{i}(t)-h_{i-1}(t)\right) /\left|h_{i}(t)-h_{i-1}(t)\right|, i=1, \ldots, \ell-1 .
$$

Proof. If $h_{i}$ 's are Lipschitz on $[0, \infty)$ and smooth except finitely many points, $u$ given by (4.10) fulfills the regularity assumptions of Theorem 3.1. Then by Theorem 3.1 and Lemma $4.2 h_{i}$ must solve (4.11) until the first merging time when $h_{i}=h_{i+1}$ for some $i$.

Of course, (4.11) is uniquely solvable until the first merging time. If $h_{i}$ 's merges at some time $t_{0}$, we removes some $x_{i}$ 's and renumber jumps $x_{i}$ 's such that $h_{i}\left(t_{0}\right) \neq$ $h_{i+1}\left(t_{0}\right)$ for $i=0,1, \ldots, \ell_{0}-2$ with $\ell_{0}<\ell$, Again we are able to solves (4.11). Repeating this procedure finitely many times, one is able to solve (4.1)-(4.2) uniquely and globally-in-time. (Since $h_{i}$ 's are bounded, the solution of (4.11) can be extended unless some $h_{i}$ 's merge.) Since the right hand side of (4.11) is bounded (independent of $t$ ), the solution $h_{i}$ 's must be globally Lipschitz continuous in time.

4.4. Constrained gradient system of ordinary differential equations. If $u=u(x, t)$ is of the form (4.10), then

$$
\begin{aligned}
& \varphi(u(t))=\psi\left(h_{1}(t), \ldots, h_{d}(t)\right), d=\ell-2(\ell \geq 3) \\
& \psi\left(h_{1}, \ldots, h_{d}\right)=\sum_{j=1}^{d+1}\left|h_{j}-h_{j-1}\right|, h_{0}=g\left(z_{0}\right), h_{\ell-1}=g\left(z_{1}\right) .
\end{aligned}
$$

(If $\ell=2, \varphi(u(t))=\left|h_{1}-h_{0}\right|$ and is independent of $t$.) Using this $\psi: \mathbf{R}^{N d} \rightarrow \mathbf{R}$, we are able to rewrite (4.11) as

$$
\frac{d h}{d t}=-\pi_{h} \operatorname{grad}_{*} \psi(h), h(t)=\left(h_{1}(t), \ldots, h_{d}(t)\right),
$$

where $\operatorname{grad}_{*}$ is the gradient of $\psi$ in $\mathbf{R}^{N d}$ with respect to the inner product

$$
(h, g)_{*}=\sum_{i=1}^{d} L_{i}\left(h_{i}, g_{i}\right)
$$


for $g=\left(g_{1}, \ldots, g_{d}\right)$ and $\pi_{h}=\left(\pi_{h_{1}}, \ldots, \pi_{h_{d}}\right)$. Indeed, by definition,

$$
\operatorname{grad}_{*} \psi(h)=\left(L_{i}^{-1} \frac{\partial \psi}{\partial h_{i}}\right)_{i=1}^{d}
$$

Since $\frac{\partial \psi}{\partial h_{i}}(t)=-\left(m_{i+1}(t)-m_{i}(t)\right),(4.11)$ is the same as (4.13). This weight is very natural since our subdifferential of $\varphi$ is taken with respect to $L^{2}(\Omega)$-inner product. Let us summarize what we obtained here.

Proposition 4.4. Assume that $M$ is compact. Let $h(t)$ be a function defined in Theorem 4.3. Then $h$ solves (4.13) for $t$ before the first merging time.

We expect that in finite time our solution $u$ stops moving. We shall prove such a phenomena when $M=S^{1}$. For this purpose we study the large time behavior of (4.13) assuming that there is no merging of $h_{i}$ 's.

Proposition 4.5 Assume that $M$ is compact. Let $h$ be a global solution of (4.13) for $t \in\left[t_{*}, \infty\right)$ such that no $h_{i}$ 's merge for $t \in\left[t_{*}, \infty\right)$. Then

$$
\int_{t_{0}}^{\infty}\left(h_{t}, h_{t}\right)_{*} d t \leq \psi\left(h\left(t_{*}\right)\right) \quad \text { and } \quad \frac{d \psi(h(t))}{d t} \leq 0 \quad \text { for } \quad t>t_{*} .
$$

Moreover, there is a subsequence of $\left\{u\left(x, t+t_{*}+k\right)\right\}_{k=1}^{\infty}$ converges in $L^{2}(\Omega \times$ $(0,1) ; M)$ to a piecewise constant stationary solution $u_{\infty}$ of $(4.1)$ in the sense that ${ }^{0} P_{u_{\infty}}\left(\partial \varphi\left(u_{\infty}\right)\right)=0$. Here $u(x, t)$ is defined by (4.12).

Proof. We observe that $h$ is smooth for $\left(t_{*}, \infty\right)$. We take inner product of $(4.13)$ and $h_{t}$ and observe that

$$
\left(h_{t}, h_{t}\right)_{*}=-\frac{d \psi}{d t}(h(t))
$$

which yields $d \psi(h(t)) / d t \leq 0$ for all $t \in\left(t_{*}, \infty\right)$. We integrate over $\left(t_{*}, s\right)$ and send $s$ to infinity to get

$$
\int_{t_{*}}^{\infty}\left(h_{t}, h_{t}\right)_{*} d t \leq \psi\left(h\left(t_{*}\right)\right)
$$

since $\psi \geq 0$. In particular, $\left(h_{k}\right)_{t}(t)=h_{t}\left(t+t_{k}+k\right)$ converges in $L^{2}(0,1)$ to zero. Since $\left\{h_{k}(t)\right\} \subset M$ is bounded for $t \in(0,1]\left\{h_{k}(t)\right\}$ has a convergent subsequence. Since $\left(h_{k}\right)_{t} \rightarrow 0$ in $L^{2}(0,1)$, the limit of $\left\{u_{k}\right\}$ (defined by (4.10) with $h_{i}$ replaced by $h_{k i}$ ) converges to $u_{\infty}$ (by taking a subsequence) which is a stationary solution. (In this argument there might be a chance that $\left(h_{i}-h_{i-1}\right)(t) \rightarrow 0$ as $t \rightarrow \infty$ so we rather use $u$ instead of $h$ ).

4.5. $S^{1}$-valued problem. We shall study a more detailed dynamics when the set of constraint $M$ equals the unit circle $S^{1}$ in $\mathbf{R}^{2}$. We first characterize all stationary piecewise constant solutions. For two vectors in $p, q \in M$ we define $\arg (p, q)=$ $\arg p-\arg q$. The value is taken so that $\arg (p, q) \in(-\pi, \pi]$.

LEMma 4.6. Let $u_{0}$ be of the form (4.2) with $h_{i}^{0} \neq h_{i+1}^{0}$ for $i=0,1, \ldots \ell-2, \ell \geq 2$ and $h_{0}^{0}=g\left(z_{0}\right)$ and $h_{\ell-1}^{0}=g\left(z_{1}\right)$. Then $u_{0}$ is a stationary solution of (4.1) (in the 
sense that $\left.{ }^{0} P_{u_{0}}\left(\partial \varphi\left(u_{0}\right)\right)=0\right)$ if and only if $\arg \left(h_{i}^{0}, h_{i-1}^{0}\right)$ is independent of $i=$ $1,2, \ldots, \ell-1$.

Proof. We may assume $\ell \geq 3$. By elementary geometry we observe that

$$
\pi_{h_{i}^{0}}\left(m_{i+1}^{0}-m_{i}^{0}\right)=0
$$

is equivalent to say that $\arg \left(h_{i}^{0}, h_{i-1}^{0}\right)=\arg \left(h_{i+1}^{0}, h_{i}^{0}\right)$ for $i=1, \ldots, \ell-2$.

We next study the stability of stationary solutions. For $u_{0}$ in (4.2) we observe that

$$
\varphi\left(u_{0}\right)=\sum_{i=1}^{\ell-1}\left|h_{i}^{0}-h_{i-1}^{0}\right|=\sum_{i=1}^{\ell-1} 2\left|\sin \xi_{i}\right|, \quad \xi_{i}=\frac{1}{2} \arg \left(h_{i}^{0}, h_{i-1}^{0}\right) .
$$

Since $h_{0}^{0}$ and $h_{\ell-1}^{0}$ are fixed by the Dirichlet data, the sum $\sum_{i=1}^{\ell-1} \xi_{i}=: \lambda$ is constant independent of $\left(\xi_{1} \ldots, \xi_{\ell-1}\right)$ (at least small perturbation of $\left(\xi_{1}, \ldots, \xi_{d}\right)$ ). We set $E\left(\xi_{1}, \ldots, \xi_{d}\right)=\sum_{i=1}^{d-1}\left|\sin \xi_{i}\right|+\left|\sin \left(\lambda-\sum_{j=1}^{d} \xi_{j}\right)\right|, d=\ell-2$. By definition $E\left(\xi_{1}, \ldots, \xi_{d}\right)=\varphi\left(u_{0}\right) / 2$. If $u_{0}$ is a stationary solution of (4.1), then by Lemma 4.6 we see that $\xi_{1}=\xi_{2}=\cdots=\xi_{d}=\lambda-\sum_{i=1}^{d} \xi_{i}$. The next lemma shows that such a stationary solution is local maximum of $E$ so in particular it is unstable in all direction. Note that when we discuss the stability it suffices to check Hesse matrix for $\operatorname{grad}(=\nabla)$ instead of $\operatorname{grad}_{*}$.

Lemma 4.7. Assume that $d=\ell-2 \geq 1$. Assume that $\lambda \neq 0$ and $\lambda /(\ell-1) \in$ $(-\pi / 2, \pi / 2]$. Then the Hesse matrix $\nabla^{2} E$ at $\xi_{0}=(\lambda /(\ell-1), \ldots, \lambda /(\ell-1))$ is negative definite.

Proof. We may assume that $\lambda>0$. We differentiate $E$ and observe that

$$
\begin{aligned}
& \nabla E=\left(\cos \xi_{i}-\cos \left(\lambda-\sum_{j=1}^{d} \xi_{j}\right)\right)_{i=1}^{d} \quad \text { near } \quad \xi_{0} \quad \text { and } \\
& -\nabla^{2} E\left(\xi_{0}\right)=\left(\delta_{i j} a+a\right)_{1 \leq i, j \leq d}, a=\sin (\lambda /(\ell-1)),
\end{aligned}
$$

where $\delta_{i j}$ is Kronecker's delta. Since

$$
\left(\delta_{i j} a+a\right)=a\left(\delta_{i j}+\sigma_{i} \sigma_{j}\right) \quad \text { with } \quad \sigma=\left(\sigma_{1}, \ldots, \sigma_{d}\right)=(1, \ldots, 1),
$$

its determinant is easy to calculate. Indeed,

$$
\operatorname{det}\left(\delta_{i j} a+a\right)=a^{d} \operatorname{det}\left(\delta_{i j}+\sigma_{i} \sigma_{j}\right)=a^{d}\left(1+|\sigma|^{2}\right)=a^{d}(1+d) .
$$

Thus we conclude that

$$
\operatorname{det}\left(\left(\delta_{i j} a+a\right)_{1 \leq i, j \leq r}\right)>0
$$

for all $r=1,2, \ldots, d$, which implies that $-\nabla^{2} E\left(\xi_{0}\right)$ is positive definite.

By Lemma 4.7 all piecewise constant stationary solution (except one jump or no jump solution) are local maximum in a class of piecewise constant functions having the same location of jump discontinuities. Of course all one jump and no jump 
solutions are isolated global minimizers since each stationary solution has a different value of energy $\varphi$. Combining Proposition 4.5 and Lemmas 4.6, 4.7, we obtain a full convergence result.

Proposition 4.8. Assume that $M=S^{1}$ and $N=2$. Let $u$ be of the form (4.10) and $h=\left(h_{1}, \ldots, h_{\ell-2}\right)$ solves (4.13) for $t \in\left[t_{*}, \infty\right)$ such that no $h_{i}$ 's merges for $t \in\left[t_{*}, \infty\right)$. Assume that $u\left(x, t_{*}\right)$ is not a stationary solution of (4.1). Then $u(x, t)$ converges to a (piecewise constant) stationary solution with jump discontinuities strictly contained in $\left\{x_{i}\right\}_{i=1}^{\ell-1}$. In particular, $h_{i}-h_{i-1} \rightarrow 0$ as $t \rightarrow \infty$ for some $i=1, \ldots, \ell-1$, as $t \rightarrow \infty$.

4.6. Stopping in finite time. We continue to study the case when $M=S^{1}$ with $N=2$. We shall prove that our piecewise constant solution $u=u(t)$ actually stops moving after finite time and it becomes a stationary solution. For this purpose we shall rewrite (4.11) by using argument $\theta_{i}(t)$ of $h_{i}(t)$. Since

$$
\begin{aligned}
m_{i+1} & =\left(\cos \theta_{i+1}-1, \sin \theta_{i+1}\right) / A_{i+1}, \\
m_{i} & =\left(1-\cos \theta_{i-1},-\sin \theta_{i-1}\right) / A_{i-1}
\end{aligned}
$$

with $A_{i}=\left(\left(\cos \theta_{i}-1\right)^{2}+\sin ^{2} \theta_{i}\right)^{\frac{1}{2}}$ if $h_{i}=(1,0)$, we see that

$$
\pi_{h_{i}}\left(m_{i+1}-m_{i}\right)=\tau\left(\sin \theta_{i+1} / A_{i+1}+\sin \theta_{i-1} / A_{i-1}\right)
$$

with $\tau=(0,1)$. Since $A_{i}^{2}=4 \sin ^{2}\left(\theta_{i} / 2\right)$, we see that

$$
\pi_{h_{i}}\left(m_{i+1}-m_{i}\right)=\tau\left(\frac{\sin \theta_{i+1}}{2\left|\sin \left(\theta_{i+1} / 2\right)\right|}+\frac{\sin \theta_{i-1}}{2\left|\sin \left(\theta_{i-1} / 2\right)\right|}\right) .
$$

For a general $h_{i}=\left(\cos \theta_{i}, \sin \theta_{i}\right)$ our calculation shows that

$$
\begin{aligned}
\pi_{h_{i}}\left(m_{i+1}-m_{i}\right)= & \tau\left\{\frac{\sin \left(\theta_{i+1}-\theta_{i}\right)}{2\left|\sin \left(\frac{\theta_{i+1}-\theta_{i}}{2}\right)\right|}+\frac{\sin \left(\theta_{i-1}-\theta_{i}\right)}{2\left|\sin \left(\frac{\theta_{i-1}-\theta_{i}}{2}\right)\right|}\right\} \\
= & \tau\left\{\operatorname{sgn}\left(\sin \frac{\theta_{i+1}-\theta_{i}}{2}\right) \cos \frac{\theta_{i+1}-\theta_{i}}{2}\right. \\
& \left.+\operatorname{sgn}\left(\sin \frac{\theta_{i-1}-\theta_{i}}{2}\right) \cos \frac{\theta_{i-1}-\theta_{i}}{2}\right\}
\end{aligned}
$$

with $\tau=\left(-\sin \theta_{i}, \cos \theta_{i}\right)$. Since

$$
\frac{d h_{i}}{d t}=\tau \frac{d \theta_{i}}{d t}
$$

(4.11) becomes

$$
\begin{aligned}
\frac{d \theta_{i}}{d t}= & L_{i}^{-1}\left[\operatorname{sgn}\left(\sin \frac{\theta_{i+1}-\theta_{i}}{2}\right) \cos \frac{\theta_{i+1}-\theta_{i}}{2}\right. \\
& \left.+\operatorname{sgn}\left(\sin \frac{\theta_{i-1}-\theta_{i}}{2}\right) \cos \frac{\theta_{i-1}-\theta_{i}}{2}\right]
\end{aligned}
$$

for $i=1, \ldots, \ell-2$. If we consider the evolution of $u,(4.14)$ holds until the first merging times of $h_{i}$ 's. At the merging time we renumber jumps so that renumbered $\theta_{i}$ 's solves (4.14) until the next merging time. We set $\xi_{i}=\left(\theta_{i}-\theta_{i-1}\right) / 2$ as before. 
Theorem 4.9 (Stopping In finite time). Assume that $N=2$ and $M=S^{1}$. Let $u$ be the solution of (4.1)-(4.2) of the form (4.10). Then there exists $t_{*} \geq 0$ such that $u(x, t)=U(x)$ for $t \geq t_{*}$ with some (piecewise constant) stationary solution of (4.1).

Proof. We may assume that the initial data is not a stationary solution. Then there are finitely many times $t_{0}<t_{1}<\cdots<t_{s}, t_{0}>0$ such that the set of jump discontinuities decreases at $t_{j}, j=0, \ldots, s$ while in $\left[0, t_{0}\right),\left[t_{j}, t_{j+1}\right),(j=0, \ldots, s-1)$ and $\left[t_{s}, \infty\right)$ the set of jump discontinuities is independent of time. (At each $t_{j}$ some $h_{i}$ merges.) We claim that $u\left(x, t_{s}\right)=U(x)$ - some stationary solution so that $u(x, t)=$ $U(x)$ for $t>t_{s}$. If $u\left(x, t_{s}\right)$ is not a stationary solution, then we have a situation of Proposition 4.8 with $t_{*}=t_{s}$. By Proposition 4.8 there exists an nonempty set $I \subset \Lambda=\{1, \ldots, \ell-1\}$ that satisfies

$$
\lim _{t \rightarrow \infty}\left(\theta_{i}(t)-\theta_{i-1}(t)\right)=0 \quad \text { for } \quad i \in I .
$$

(i)If $I \neq \Lambda$, then there is $i_{0} \in I$ such that either $i_{0}+1$ or $i_{0}-1$ does not belong to $I$. If $i_{0}+1 \notin I$, then $\left|d \theta_{i_{0}} / d t\right|$ is bounded away from zero for sufficiently large $t$ by (4.14) since $\theta_{i_{0}}-\theta_{i_{0}-1} \rightarrow 0$ while $\theta_{i_{0}+1}-\theta_{i_{0}}$ is bounded away from zero. Similarly, if $i_{0}-1 \notin I$ then $\left|d \theta_{i_{0}-1} / d t\right|$ is bounded away from zero for sufficiently large $t$. In both cases these properties contradict the convergence of $h_{i_{0}}$ or $h_{i_{0-1}}$ as $t \rightarrow \infty$. So this case does not occur.

(ii)If $I=\Lambda$, then $g\left(z_{0}\right)=g\left(z_{1}\right)$. Then there is some $i_{0} \in \Lambda$ such that $\operatorname{sgn} \sin \xi_{i_{0}}>0$ and either $\operatorname{sgn} \sin \xi_{i_{0}+1}>0$ or $\operatorname{sgn} \sin \xi_{i_{0}-1}>0$. Note that $\operatorname{sgn} \sin \xi_{i_{0}+1}(t)$ is independent of $t \geq t_{*}$. By (4.14) either $\left|d \theta_{i_{0}} / d t\right|$ or $\left|d \theta_{i_{0}-1} / d t\right|$ is bounded away from zero for large $t$. This property contradicts the convergence of $h_{i_{0}}$ or $h_{i_{0}-1}$ as $t \rightarrow \infty$. So this case does not occur neither.

We thus conclude that $u\left(x, t_{s}\right)=U(x)$.

REMARK 4.10. The stationary solution $U(x)$ we obtain at $t_{s}$ is not necessarily one jump or no jump solution. Here is a simple example. We set

$$
h_{0}^{0}=(0,-1), h_{3}^{0}=(0,1), h_{1}^{0}=\left(\cos \theta_{0}, \sin \theta_{0}\right), h_{2}^{0}=\left(\cos \theta_{0},-\sin \theta_{0}\right)
$$

with $\ell=4$ and $\theta_{0} \in(0, \pi / 2)$. Assume that the initial data $u_{0}$ is given by (4.2) with these $h_{i}^{0}$ 's and that $L_{0}=L_{1}=L_{2}=L_{3}$. Then the solution $u(x, t)$ becomes

$$
U(x)= \begin{cases}h_{0}^{0}, & x \in\left(x_{0}, x_{1}\right), \\ (1,0), & x \in\left(x_{1}, x_{3}\right), \\ h_{3}^{0}, & x \in\left(x_{3}, x_{4}\right)\end{cases}
$$

at the first merging time which is a stationary solution.

Although all piecewise constant stationary solution (except one or no jump solution) are local maximum in a class of piecewise constant functions having the same location of jump discontinuities, it may be attained at the merging time of evolution as this example shows. 


\section{Numerics and simulations.}

5.1. Numerical scheme. In this section we concentrate on the special situation where $n=1, N=2$ and $M=S^{1}$ as was argued in the last part of the previous section. We assume $\Omega=(0,1)$ for simplicity, and consider the angle variable $\theta=\theta(x)$ whose values are restricted to the interval $(-\pi, \pi]$. Using this variable we formally rewrite the energy functional (1.1) as follows,

$$
E[\theta]=\int_{0}^{1}\left[|\nabla \cos \theta|^{2}+|\nabla \sin \theta|^{2}\right]^{\frac{1}{2}} d x .
$$

Although (5.1) seems to be deformed to the simpler form

$$
E[\theta]=\int_{0}^{1}|\nabla \theta| d x
$$

by the formal calculation $\nabla \cos \theta=-\sin \theta \cdot \nabla \theta$ and $\nabla \sin \theta=\cos \theta \cdot \nabla \theta$, we do not adopt (5.2) because this cannot count the energy correctly when the discontinuity exists. The energy (5.1) measures the variation by the natural metric in $R^{2}$-space where $S^{1}$ is embedded, while (5.2) sums up the variation along the arc of $S^{1}$. The energy (1.1) does require the former type (5.1) of measurement. Note that (5.1) and (5.2) are the same as long as $\theta(x)$ is continuous.

In order to construct our numerical scheme, we first discretize the energy functional (5.1) in the space variable. Let us approximate an arbitrary function by the piecewise constant function expressed in the form

$$
\theta(x)=\theta_{i} \quad \text { for } x \in\left(x_{i-1}, x_{i}\right) \quad(i=1,2, \ldots, I)
$$

where $x_{i}=i \delta x(i=0,1, \ldots, I)$ and $\delta x=1 / I$. For such functions, we define an energy functional

$$
E[\theta]=\sum_{i=2}^{I}\left[\left(\cos \theta_{i}-\cos \theta_{i-1}\right)^{2}+\left(\sin \theta_{i}-\sin \theta_{i-1}\right)^{2}\right]^{\frac{1}{2}},
$$

by evaluating the variations across the jump points. Note that it is equivalent to

$$
E[\theta]=\delta x \sum_{i=2}^{I}\left[\left(\frac{\cos \theta_{i}-\cos \theta_{i-1}}{\delta x}\right)^{2}+\left(\frac{\sin \theta_{i}-\sin \theta_{i-1}}{\delta x}\right)^{2}\right]^{\frac{1}{2}} .
$$

which is a formal approximation of the energy form (5.1). The partial derivative with respect to $\theta_{i}$ is easily obtained as follows,

$$
\frac{\partial E}{\partial \theta_{i}}=\frac{\sin \left(\theta_{i}-\theta_{i-1}\right)}{R_{i}}-\frac{\sin \left(\theta_{i+1}-\theta_{i}\right)}{R_{i+1}},
$$

where $R_{i}=\left[\left(\cos \theta_{i}-\cos \theta_{i-1}\right)^{2}+\left(\sin \theta_{i}-\sin \theta_{i-1}\right)^{2}\right]^{\frac{1}{2}}$. Using this formula, we obtain a formal expression of the gradient system

$$
\delta x \frac{d \theta_{i}}{d t}=\frac{\partial E}{\partial \theta_{i}}
$$

thus

$$
\frac{d \theta_{i}}{d t}=\frac{1}{\delta x}\left[\frac{\sin \left(\theta_{i+1}-\theta_{i}\right)}{R_{i+1}}-\frac{\sin \left(\theta_{i}-\theta_{i-1}\right)}{R_{i}}\right] \quad(i=2, \ldots, I-1)
$$


Note that $\theta_{1}$ and $\theta_{I}$ are fixed to some values since we adopt the Dirichlet boundary conditions in this paper, and the modification to Neumann boundary condition is straightforward.

Although the formula (5.3) is quite similar to (4.14), there is an essential difference between them. The equation system (4.14) is not an usual ordinary equation system, but a reducing ODE system which means that the dimension of phase space (the number of $\theta_{i}$ 's) reduces when the height of the adjacent plateaus coincide as time passes. In this process the plateaus join and increase their sizes, thus (4.14) includes the sizes $L_{i}$ 's in its expression. On the other hand, in the equation system (5.3) the number of small intervals $I$ (i.e. the number of variables) does not change and their size is always $\delta x$ even after the merging of plateaus. The equation system (5.3) is designed to solve the equation (1.5) numerically as a PDE system whose initial data might be other type of functions than piecewise constant ones as will be shown in Fig.5 and Fig.6.

Now let us give a numerical scheme using the equations (5.3) by discretizing the time variable $t$ with the uniform mesh size $\delta t$. Numerical solution is denoted by $\theta_{i}^{n}$ which expresses a value of $\theta$ in the interval $\left(x_{i-1}, x_{i}\right)$ and at the time $t^{n}=$ $n \delta t$. Singular diffusivity (i.e. infinitely large diffusivity) is replaced by the very large diffusivity using the method proposed in $[\mathrm{KG}]$. In addition the sine function is rewritten as shown below in order to design an implicit scheme yielding the linear equation system. We propose a numerical scheme as follows.

$$
\begin{aligned}
\frac{\theta_{i}^{n}-\theta_{i}^{n-1}}{\delta t}= & \frac{1}{\delta x}\left[\chi_{\gamma}\left(R_{i+1}^{n-1}\right) f\left(\theta_{i+1}^{n-1}-\theta_{i}^{n-1}-2 \pi \sigma_{i+1}^{n-1}\right)\left(\theta_{i+1}^{n}-\theta_{i}^{n}-2 \pi \sigma_{i+1}^{n-1}\right)\right. \\
& \left.-\chi_{\gamma}\left(R_{i}^{n-1}\right) f\left(\theta_{i}^{n-1}-\theta_{i-1}^{n-1}-2 \pi \sigma_{i}^{n-1}\right)\left(\theta_{i}^{n}-\theta_{i-1}^{n}-2 \pi \sigma_{i}^{n-1}\right)\right]
\end{aligned}
$$

where $\chi_{\gamma}$ is a cut-off inverse function defined by

$$
\chi_{\gamma}(\xi)=\left\{\begin{array}{cl}
\gamma & 0 \leq \xi<1 / \gamma \\
1 / \xi & 1 / \gamma \leq \xi
\end{array}\right.
$$

with a large positive number $\gamma$, and $f(\xi)=\sin \xi / \xi$. The integer $\sigma_{i}^{n-1}=0, \pm 1$ is taken so that the relation $\left|\theta_{i}^{n-1}-\theta_{i-1}^{n-1}-2 \pi \sigma_{i}^{n-1}\right| \leq \pi$ holds. The notation $R_{i}^{n-1}$ indicates $R_{i}$ at the time step $t^{n-1}$.

We obtain the linear equation system to be numerically solved as follows,

$$
-A_{i} \theta_{i-1}^{n}+B_{i} \theta_{i}^{n}-C_{i} \theta_{i+1}^{n}=D_{i}
$$

where

$$
\begin{aligned}
A_{i} & =\frac{1}{\delta x} \chi_{\gamma}\left(R_{i}^{n-1}\right) f\left(\theta_{i}^{n-1}-\theta_{i-1}^{n-1}-2 \pi \sigma_{i}^{n-1}\right), \\
C_{i} & =\frac{1}{\delta x} \chi_{\gamma}\left(R_{i+1}^{n-1}\right) f\left(\theta_{i+1}^{n-1}-\theta_{i}^{n-1}-2 \pi \sigma_{i+1}^{n-1}\right), \\
B_{i} & =\frac{1}{\delta t}+A_{i}+C_{i}, \\
D_{i} & =\frac{1}{\delta t} \theta_{i}^{n-1}+2 \pi \sigma_{i}^{n-1} A_{i}-2 \pi \sigma_{i+1}^{n-1} C_{i} .
\end{aligned}
$$

Do not forget to make newly obtained $\theta_{i}^{n}$ 's lie in the interval $(-\pi, \pi]$ by adding appropriate corrections $2 \pi \times$ (integer) on every time step. 
5.2. Simulations. Several simulations are performed using the numerical scheme (5.4), and we mostly handle the situation which was analyzed in the section 4 whose initial data is a piecewise constant functions (thus the solution is piecewise constant all through the evolution process). In the last part, two examples with the smooth initial data will be presented. Before going to demonstrate our numerical results, we give explanations of the panels (a)-(d) in Fig.1 - Fig.4. The panel (a) shows a profile of the initial data which is a piecewise constant function. Positive integers are given as indices of the plateaus in the initial data. The panel (b) is a plot of the initial data in the $u$-plane. Large white makers express the plateaus which are movable along the unit circle, while small black ones correspond to the plateaus which are fixed to the points determined by the Dirichlet conditions. The final data is presented in the panel (c) in the same way with the panel (b). The panel (d) is a graph of the orbits of the plateaus' evolution, where each number on the orbit represents the corresponding initial plateau. The notation, for example, $2+3$ means that the initial plateaus with the indices 2 and 3 merged at some time and act as a one plateau at that moment. The computational parameters are taken as follows in all the simulations; $I=400(\delta x=0.0025), \gamma=1.0 \times 10^{5}$ and $\delta t=5.0 \times 10^{-4}$.

Fig.1 shows a simple example in which only one plateau is movable and the exact solution is explicitly calculated using (4.14). By setting the angle of the central plateau $\theta(t)$ we have an ODE

$$
L \frac{d \theta}{d t}=-2 \operatorname{sgn}\left(\sin \frac{\theta}{2}\right) \cos \frac{\theta}{2}
$$

for the initial angle $\theta^{0}(\neq 0, \pi)$. It is clear that both of $\theta \equiv 0$ and $\theta \equiv \pi$ are stationary solutions and the former is stable while the latter is unstable. The exact solution of (5.5) is obtained in the inverse function form

$$
t=\frac{L}{2}\left|\log \frac{1+\sin \frac{\theta}{2}}{1-\sin \frac{\theta}{2}}-\log \frac{1+\sin \frac{\theta^{0}}{2}}{1-\sin \frac{\theta^{0}}{2}}\right| .
$$

The final state $\theta=0$ is attained at the time $t_{*}=\frac{L}{2}\left|\log \frac{1+\sin \frac{\theta^{0}}{2}}{1-\sin \frac{\theta^{0}}{2}}\right|$. The graph of this exact solution (5.6) with $L=0.6$ and $\theta^{0}=5 \pi / 6$ is drawn in the panel (d), and it agrees with the numerical solution within the graphical resolution. The convergence property is confirmed by controlling the values of $\delta t$ and $\delta x$. Fig.2 presents another example which admits an exact solution. In this case the equation for the central plateau is

$$
L \frac{d \theta}{d t}=\sqrt{2} \operatorname{sgn}(\cos \theta) \sin \frac{\theta}{2}
$$

and its exact solution is

$$
t=\frac{L}{\sqrt{2}}\left|\log \frac{1-\cos \frac{\theta}{2}}{1+\cos \frac{\theta}{2}}-\log \frac{1-\cos \frac{\theta^{0}}{2}}{1+\cos \frac{\theta^{0}}{2}}\right| .
$$

This solution is also drawn in the panel (d) which agrees with the numerical data with high accuracy.

In Fig.3 and Fig.4, the destabilization of stationary solutions and the following evolution are presented. By the lemma 4.6, a stationary solution must have a uniform gap in $\theta$ at each jump point as long as we consider piecewise constant solutions. 
The initial data of the simulation shown in Fig.3 is slightly fluctuated from the step-like stationary solution as is described in the figure and its caption. Then the solution $\theta(x, t)$ must start to deviate from the stationary solution as the lemma 4.7 claims. Actually it does, and the plateaus sequentially join to reduce their number to the final value 2 . The whole evolution process is presented in the panel $(\mathrm{d})$. In this simulation, the only discontinuity in the final state locates at the point between the third and fourth plateau in the initial data. The position of the final discontinuity depends sensitively on the fluctuation added to the initial data. We can easily see the change of the total variation in this evolution process. The total variation of the initial data is close to $16 \sin \frac{\pi}{12}$ since all the gaps of $\theta$ at the 8 discontinuous points are approximately $\frac{\pi}{6}$, while the total variation in the final state equals to $2 \sin \frac{\pi}{3}=\sqrt{3}$. Note that the continuous solution changing linearly from $-2 \pi / 3$ to $2 \pi / 3$ is obtained in the final state if we solve the constrained diffusion equation from the same initial data.

In the simulation shown in Fig.4, the initial state is also a slightly fluctuated steplike function. The gap of $\theta$ at each jump point of the stationary solution is $4 \pi / 5$ in this case. This stationary solution turns around the origin twice exactly, and consequently the Dirichlet data at the both ends coincide as is indicated by the star shape in the panel (b). Adjacent plateaus fuse to form a new one plateau until the solution reaches the final state which is constantly $9 \pi / 10$ as presented in the panel (d). If we solve the diffusion equation with this initial data, the final state is continuous and turns around the origin twice. Actually the rotation number around the origin is conserved in the diffusion equation's case, while our equation may reduce it.

In Fig. 5 we presents an evolution of the solution whose initial data is given by the linear function with small noises (the 1st panel). It takes long for the initial smooth data to destabilize, actually the linear profile looks almost unchanged until the time $t=9.0$. However, once discontinuities become observable (the $2 \mathrm{nd}$ ), the gap at the jump point grows rapidly (the 3rd) until the solution exhibit a piecewise constant profile (the 4th). Then the solution reach the final solution which has only one jump point (the 5th).

The final simulation is given in Fig.6 whose initial data is smooth function with convex parts and concave parts (the 1st panel). Such convex or concave part is quickly flattened (the 2nd), and consequently the solution becomes monotone function with plateaus (the 3rd). Then the process drastically slow down, and the profile is kept for long time. Finally the discontinuities appear in the non-flat part (the 4th), and the solution changes to the piecewise constant function. The final state is essentially the same with the previous simulation except for the position of the jump point (the 5 th).

\section{REFERENCES}

[ABCM] F. Andreu, C. Ballester, V. Caselles and J. M. Mazón, Minimizing total variation flow, Diff. Int. Eq., 14 (2001), pp. 321-360.

[ABCM2] F. Andreu, C. Ballester, V. Caselles and J. M. Mazón, The Dirichlet problem for the total variation flow, J. Funct. Anal., 180 (2001), pp. 347-403.

[ACM] F. Andreu-Vaillo, V. Caselles and J. M. Mazón, Existence and uniqueness of a solution for a parabolic quasilinear problem for linear growth functionals with $L^{1}$ data, Math. Ann., 322 (2002), pp. 139-206.

[AG] A. B. Angenent And M. E. GuRTin, Multiphase thermomechanics with interfacial structure 2, Evolution of an isothermal interface, Arch. Rational Mech. Anal., 108 (1989), pp. 323-391. 
[Br] H. BrezIS, Operateurs Maximaux Monotone, North-Holland, Amsterdam, 1973.

[Ba] V. BARBu, Nonlinear Semigroups and Differential Equations in Banach spaces, Noordnoff, Groningen, 1976.

[BCM] G. Bellettini, V. Caselles and M. Novaga, The total variation flow in $\mathbf{R}^{N}$, J. Differential Equations., 184 (2002), pp. 475-525.

[BN] G. Bellettini AND M. NovaGA, Approximation and comparison for non-smooth anisotropic motion by mean curvature in $\mathbf{R}^{N}$, Math. Mod. Methods. Appl. Sci., 10 (2000), pp. 1-10.

[Cg] K.C. Chang, Heat flow and boundary value problem for harmonic maps, Ann. Inst. Henri Poincaré Analyse Non Linéaire, 6 (1989), pp. 363-395.

[CDY] K. C. Chang, W. Y. Ding And R. Ye, Finite time blow up of the heat flow of harmonic maps from surfaces, J. Differential Geom., 36 (1992), pp. 507-515.

[Ch] Y. CHEN, The weak solutions of the evolution problems of harmonic maps, Math. Z. 201 (1989), pp. 69-74.

[ChW] Y. CHEN AND T. WunderLI, Adaptive total variation for image restoration in $B V$ space, J. Math. Anal. Appl., 272 (2002), pp. 117-137.

[C] J. M. Conon, Nonuniqueness for the heat flow of harmonic maps, Ann. Inst. H. Poincaré, Anal. Non Linéarie, 7 (1990), pp. 335-344.

[D] E. DiBenedetto, Degenerate Parabolic Equations, Springer-Verlag, New York, 1993.

[ES] J. Eells, J. H. SAmpson, Harmonic mappings of Riemannian manifolds, Amer. J. Math., 86 (1964), pp. 109-160.

[F] A. Freire, Uniqueness for the harmonic map flow in two dimensions, Calc. Var., 3 (1995), pp. 95-105.

[FG] T. FUKUI AND Y. GIGA, Motion of a graph by nonsmooth weighted curvature, in "World Congress of Nonlinear Analysis '92", (V. Lakshmikantham, ed.), de Gruyter, Berlin, volI, 1996 , pp. $47-56$.

[GMS] M. Giaquinta, G. Modica And J. SouceK, Variational problems for maps of bounded variation with values in $S^{1}$, Calc. Var., 1 (1993), pp. 87-121.

[GG1] M.-H. Giga And Y. Giga, Generalized motion by nonlocal curvature in the plane, Arch. Rational Mech. Anal., 159 (2001), pp. 295-333.

[GG2] M.-H. GIGA AND Y. GIGA, Minimal vertical singular diffusion preventing overturning for the Burgers equation, Contem. Math., 330 (2003), pp. 73-88.

[GGK] M.-H. Giga, Y. Giga and R. KobaYAShi, Very singular diffusion equations, Proc. of Taniguchi Conf. on Math., Advanced Studies in Pure Mathematics, 31 (2001), pp. 93-125.

[G] Y. GigA, Surfaces Evolution Equations - a level set method, Hokkaido University Technical Report Series in Mathematics, \#71, Sapporo, 2002, also Lipschitz Lecture Notes 44, University of Bonn, 2002.

[GKY] Y. Giga, Y. Kashima and N. YAmazaki, Local solvability of a constrained gradient system of total variation, Abstract and Applied Analysis, to appear.

[GPR] Y. Giga, M. PAOLINI AND P. RYBKA, On the motion by singular interfacial energy, Japan J. Indust. Appl. Math., 18 (2001), pp. 231-248.

[GBP] L. GRÁNÁSY, T. BörzSÖNYI AND T. PuszTAI, Nucleation and bulk crystallization in binary phase field theory, Phys. Rev. Lett., 88 (2002), art. no. 206105.

[Gu] M. E. GuRTin, Thermomechanics of Evolving Phase Boundaries in the Plane, Oxford, Clarendon Press, 1993.

[HZ] R. HARDT AND X. ZHOU, An evolution problem for linear growth functionals, Comm. Partial Differential Equations, 19 (1994), pp. 1879-1907.

[KG] R. KobaYAShi AND Y. GIGA, Equations with singular diffusivity, J. Stat. Phys., 95 (1999), pp. 1187-1220.

[KWC] R. Kobayashi, J. A. Warren and W. C. Carter, A Continuum Model of Grain Boundaries Physica D, 140 (2000), pp. 141-150.

[Ko] Y. Kōmura, Nonlinear semi-groups in Hilbert space, J. Math. Soc. Japan, 19 (1967), pp. 493-507.

[LW] A. E. Lobkovsky AND J. A. WARRen, Sharp interface limit of a phase-field model of crystal grains, Phys. Rev. E, 63 (2001), art. no. 051605.

[MSO] F. MÉmoli, G. SAPIRo AND S. OSher, Solving variational problems and partial differential equations mapping into general target manifold, IMA Preprint Series, 1827 (2001).

[ROF] L. I. Rudin, S. Osher AND E. FAtemi, Nonlinear total variation based noise removal algorithms, Physica D, 60 (1992), pp. 259-268.

[S] G. SAPIRO, Geometric Partial Differential Equations and Image Analysis, Cambridge 
University Press, United Kingdom, 2001.

[St] M. Struwe, On the evolution of harmonic mappings of Riemannian surfaces, Comm. Math. Helvetici, 60 (1985), pp. 558-581.

[TSC] B. TANG, G. SAPIRO AND V. CASselles, Color image enhancement via chromaticity diffusion, IEEE Transactions on Image Processing, 10:5 (2001), pp. 701-707.

[T] J. TAYLOR, Constructions and conjectures in crystalline nondifferential geometry, In: Differential Geometry (eds. B. Lawson and K. Tanenblat), Proceedings of the Conference on Differential Geometry, Rio de Janeiro, Pitman Monograph Surveys Pure Appl. Math., 52 (1991), pp. 321-336.

[TGO] Y.-H. R. Tsai, Y. GIGA AND S. Osher, A level set approach for computing discontinuous solutions of a class of Hamilton-Jacobi equations, Math. Comp., 72 (2003), pp. 159181.

[WKC] J. A. Warren, R. Kobayashi and W. C. Carter, Modeling grain boundaries using a Phase-field technique, J. Cryst. Growth, 211 (2000), pp. 18-20. 


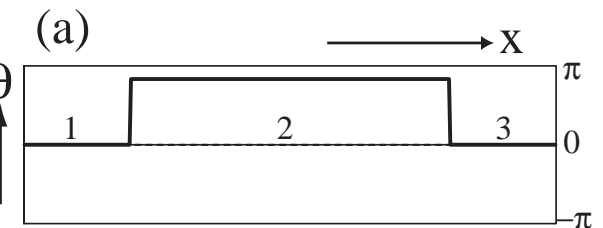

(b)

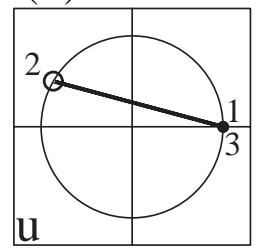

(c)

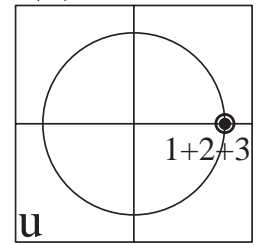

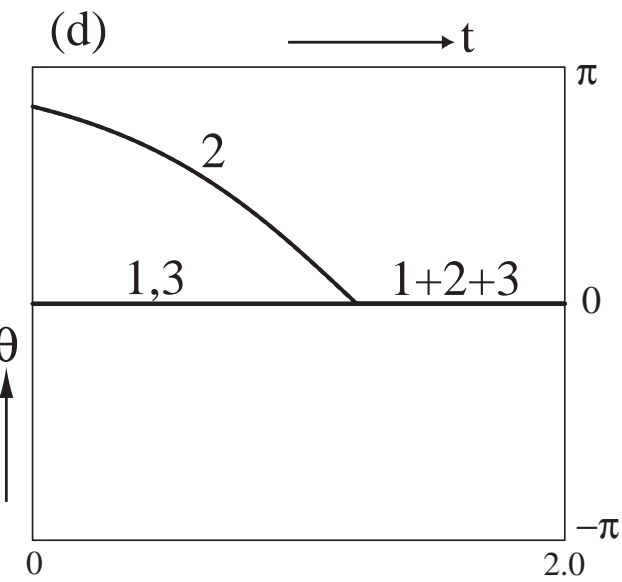

FIG. 1. Dirichlet data at the both ends are set to 0. In the initial data, the number of plateau is 3 , the value of $\theta$ is $5 \pi / 6$ in the central interval whose length is 0.6 .

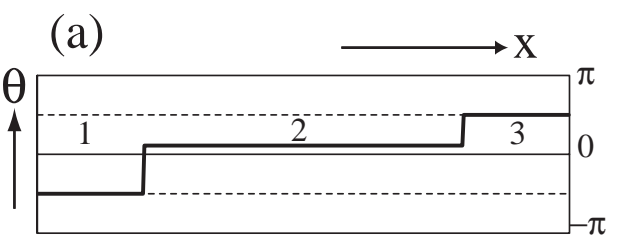

(b)

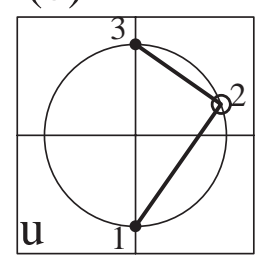

(c)

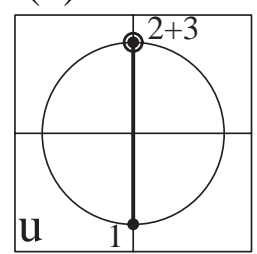

(d)

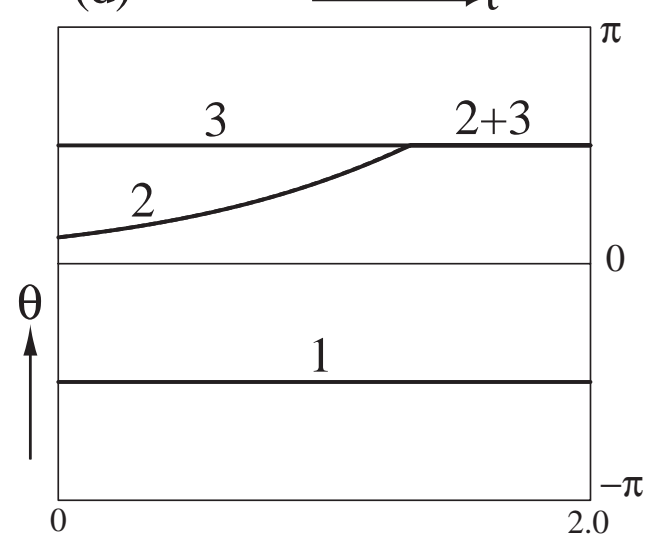

FiG. 2. Dirichlet data at the left end is set to $-\pi / 2$ and the one at the right is set to $\pi / 2$. In the initial data, the number of plateau is 3 , the value of $\theta$ is $\pi / 9$ in the central interval whose length is 0.6 . 

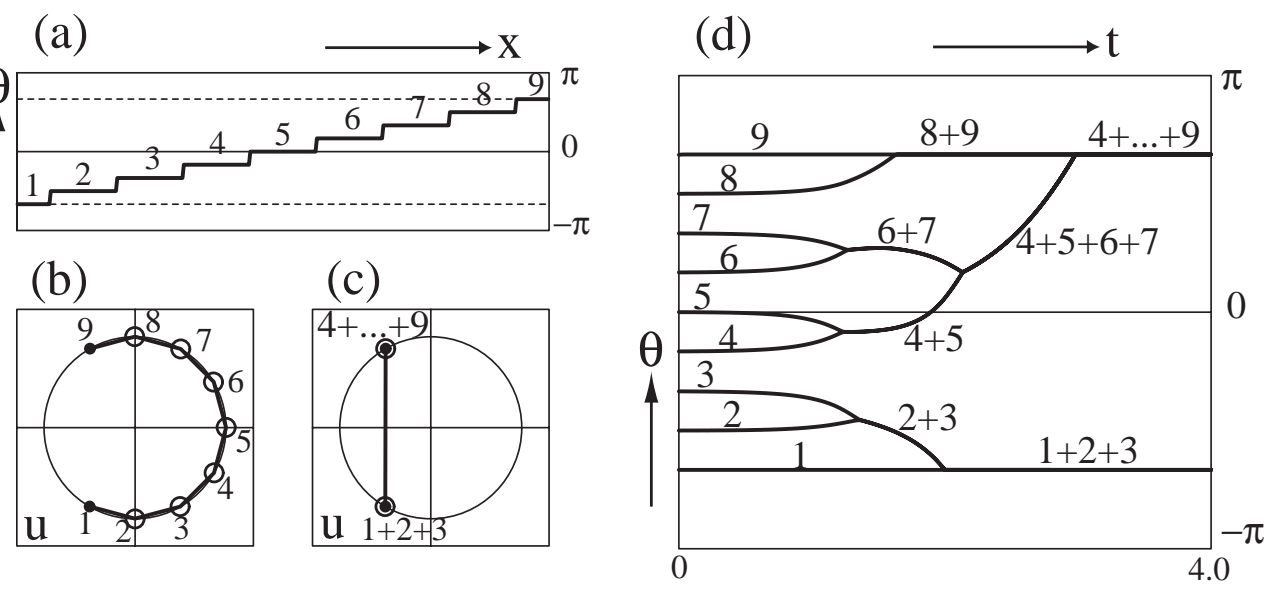

FIG. 3. Dirichlet data at the left end is set to $-2 \pi / 3$ and the one at the right is set to $2 \pi / 3$. In the initial data, the number of plateau is 9 , the value of $\theta$ is $(k-5) \pi / 6+a_{k}$ in the $k$-th interval whose length is $1 / 8$ for $k=2, \ldots, 8$ and $1 / 16$ for $k=1,9$. The sequence $\left\{a_{k}\right\}(k=2, \ldots, 8)$ is given by small random numbers.
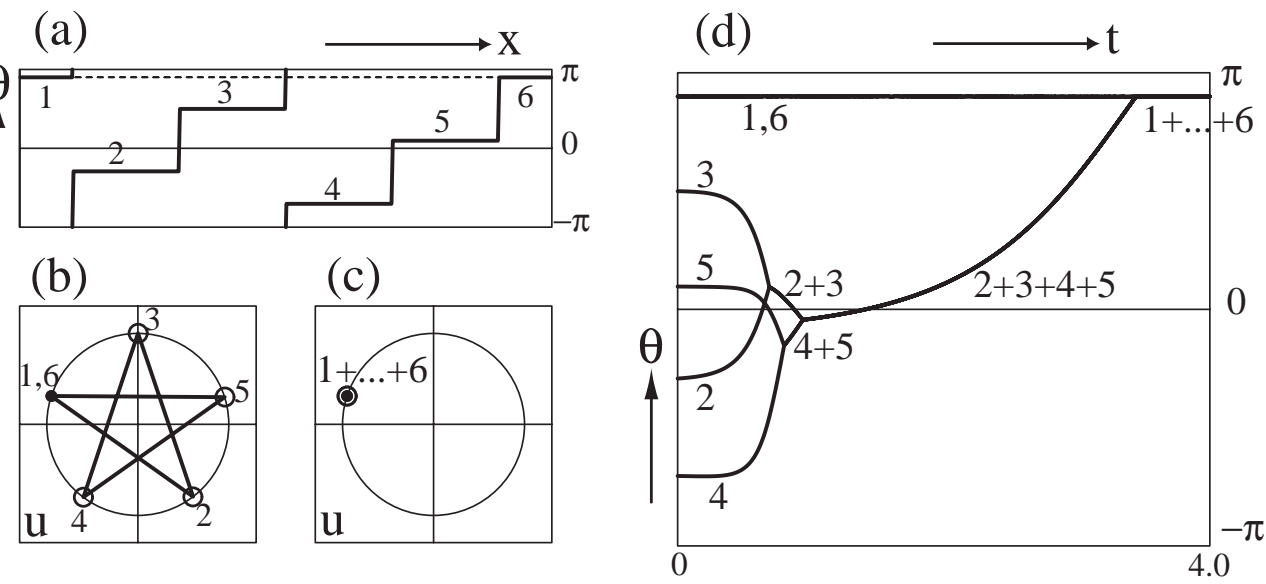

FIG. 4. Dirichlet data at the both end is set to $p \pi / 10$. In the initial data, the number of plateau is 6 , the value of $\theta$ is $(8 k+1) \pi / 10+a_{k}$ (restricted to the interval $\left.(-\pi, \pi)\right)$ in the $k$-th interval whose length is $1 / 5$ for $k=2, \ldots, 5$ and $1 / 10$ for $k=1,6$. The sequence $\left\{a_{k}\right\}(k=2, \ldots, 5)$ is given by small random numbers. 

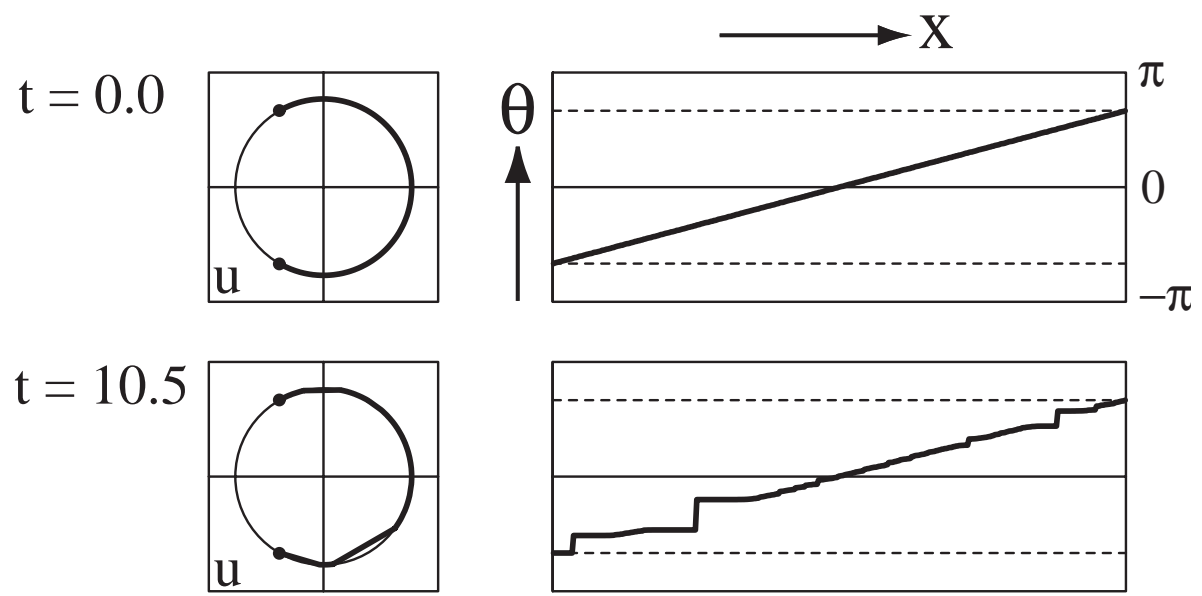

$\mathrm{t}=11.0$
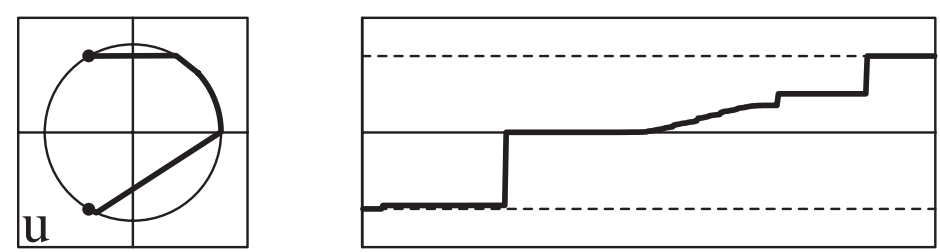

$\mathrm{t}=11.5$
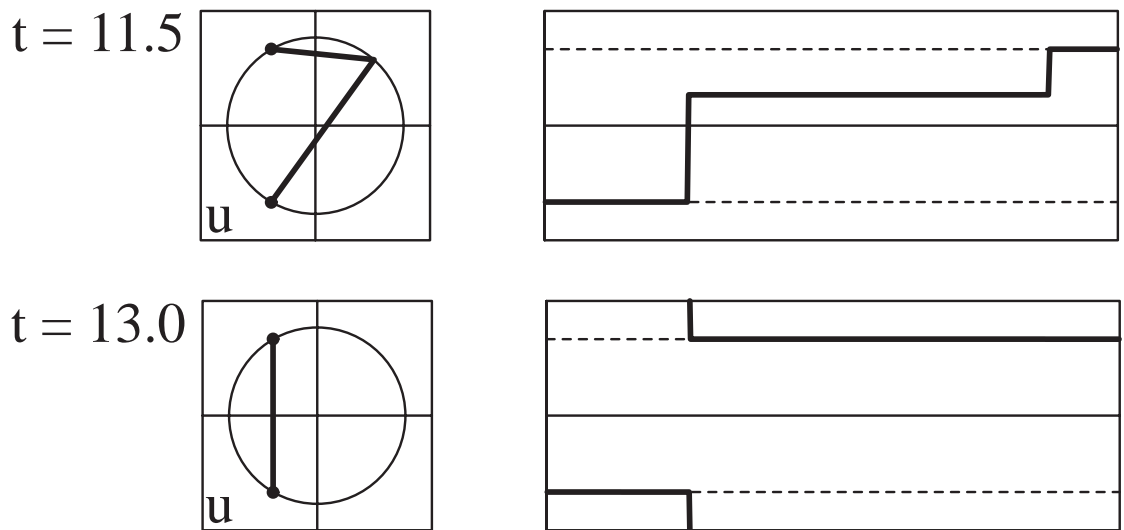

FIG. 5. Dirichlet data at the left end is set to $-2 \pi / 3$ and the one at the right is set to $2 \pi / 3$. The initial data is given by $\theta(x)=\frac{2 \pi}{3}(2 x-1)+$ noise. 

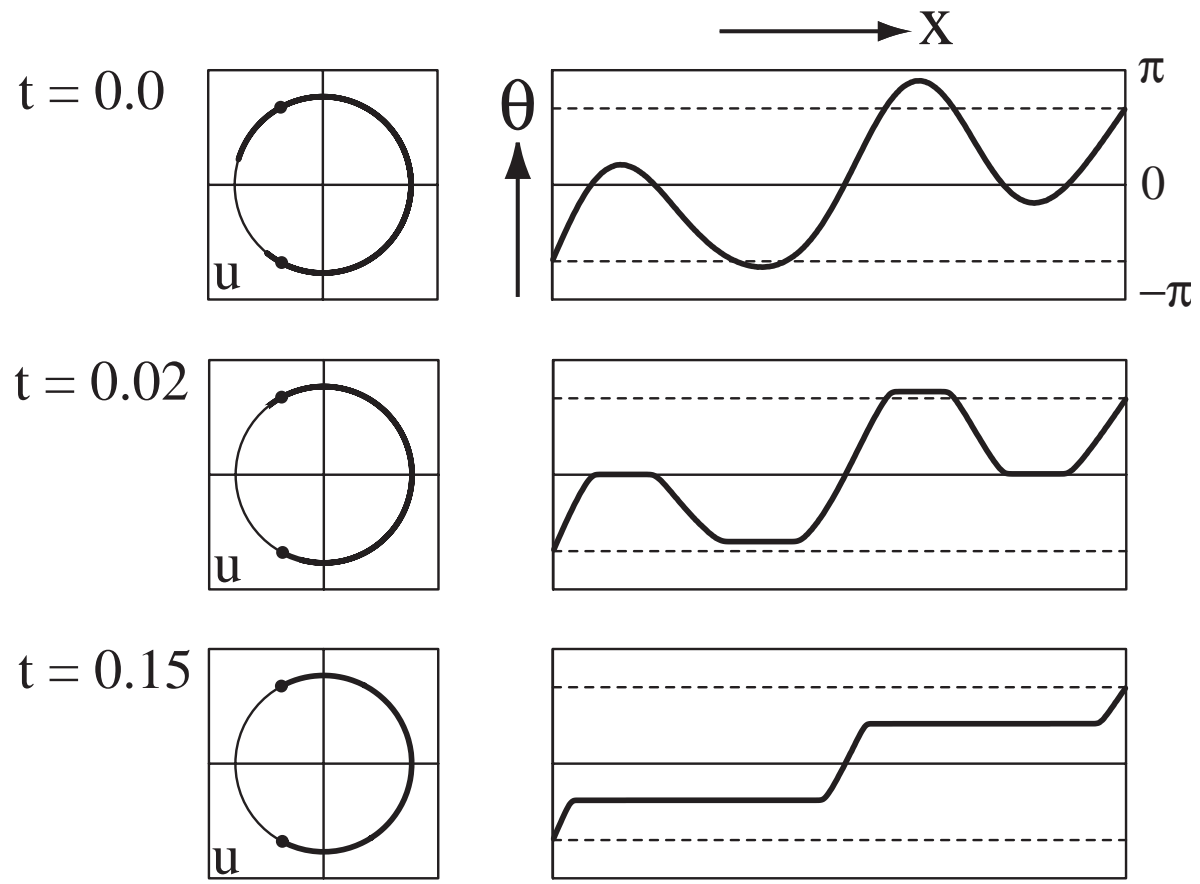

$\mathrm{t}=1.0$
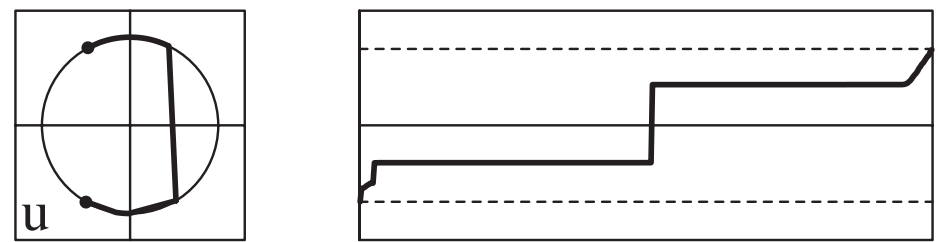

$\mathrm{t}=2.0$
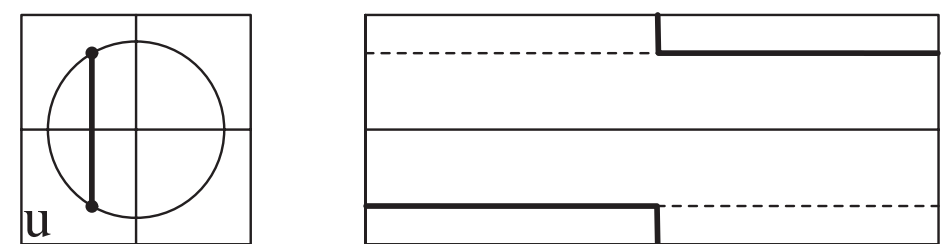

FIG. 6. Dirichlet data at the left end is set to $-2 \pi / 3$ and the one at the right is set to $2 \pi / 3$. The initial data is given by $\theta(x)=\frac{2 \pi}{3}(2 x-1)+2 \sin 4 \pi x+0.3 \sin 7 \pi x+$ noise. 
